\title{
On Performance of Full-Duplex Decode-and-Forward Relay Systems with an Optimal Power Setting under the Impact of Hardware Impairments
}

\author{
Ba Cao Nguyen $\mathbb{D}^{1,}{ }^{1,2}$ Xuan Nam Tran $\mathbb{D}^{1},{ }^{1}$ Thi Thu Hang Nguyen, ${ }^{1}$ and Dinh Tan Tran ${ }^{2}$ \\ ${ }^{1}$ Advanced Wireless Communications Group, Le Quy Don Technical University, Ha Noi, Vietnam \\ ${ }^{2}$ Telecommunications University, Khanh Hoa, Vietnam \\ Correspondence should be addressed to Xuan Nam Tran; namtx@mta.edu.vn
}

Received 14 October 2019; Revised 27 August 2020; Accepted 14 September 2020; Published 29 September 2020

Academic Editor: Donatella Darsena

Copyright ( $92020 \mathrm{Ba}$ Cao Nguyen et al. This is an open access article distributed under the Creative Commons Attribution License, which permits unrestricted use, distribution, and reproduction in any medium, provided the original work is properly cited.

\begin{abstract}
In this paper, we analyze the performance of in-band full-duplex (IBFD) relay systems that use the decode-and-forward (DF) protocol at the relay under the impact of imperfect self-interference cancellation and hardware impairments. Three practical relay scenarios are considered in our analysis: (i) there is no direct link from the source to the destination; (ii) there is a direct link, but the signal from the source is considered interference; and (iii) there is a direct link, and the signal from the source is cooperatively combined with that from the relaying path. Specifically, we derive exact and asymptotic expressions for the outage probability (OP) of the IBFD system. Based on the OP, the exact expression for symbol error probability (SEP) is also derived. Moreover, in order to cope with the effect of imperfect self-interference cancellation (SIC) due to the full-duplex mode, we propose optimal and suboptimal power calculation methods for the relay to minimize the OP and SEP. A performance evaluation shows that the IBFD relay system is significantly affected by both imperfect SIC and hardware impairments. However, the optimal power values can help to improve the system performance, significantly.
\end{abstract}

\section{Introduction}

The last decade has witnessed significant development of wireless technologies. In particular, the evolvement of the fifth generation (5G) of mobile communications allows deployment of massive Internet of Things (IoT) devices to provide data access for various applications such as precision agriculture, disaster warning, smart retail, health care, smart home, and industrial innovation. It is expected that there will be billions of IoT devices connected to the cellular networks in the coming years. The main features of the massive IoT devices include low cost, extreme coverage, narrow band, and small volume of data. In order to meet these requirements, multiple antennas are not used in the devices but wireless relaying is desired to extend the coverage. It also requires that spectrum is efficiently used to support massive connections within the limited radio frequency band.

In the literature, there were a large number of works studying the relay communications for the wireless networks.
With the help of a relay node, it is possible to extend the network range as well as increase the channel capacity. Relay communication has now found its application in various types of wireless networks such as the long-term evolution advanced (LTE-A) mobile communication, ad hoc network, and vehicle-to-vehicle (V2V) networks. Meanwhile, in-band full-duplex (IBFD) communication, which allows for simultaneous transmission and reception over the same frequency, has attracted increasing attention due to its capability to double the channel capacity and increase the spectrum efficiency. Benefits of using both IBFD and relay communications have been studied widely [1-11]. Recently, the combination of IBFD and relay cooperation has also been considered for the 5th generation (5G) heterogeneous networks (HetNets) [12].

Most studies on IBFD relay networks tackle the problem of residual self-interference (RSI) due to imperfect selfinterference cancellation (SIC) (note that this abbreviation differs from that used for successive interference cancellation 
in CDMA, NOMA, and MIMO systems) at the full-duplex relay node [2, 4, 13-15]. A paper [4] showed that the outage performance of the IBFD relay system is significantly affected by RSI. In this work, the authors have also successfully derived the closed form of the outage probability (OP) of the IBFD relay network. In [6], the authors analyzed the spectrum efficiency of the IBFD relay systems by using the sum rate as a function of the distance between users and the relay. Performance of the IBFD systems was shown to depend on the interference suppression capability and location of the relay. The work in [16] considered an IBFD multiuser decode-and-forward (DF) relay system with self-interference management. In order to maximize the signal-to-noise ratio (SNR) at the receiver, the authors in [17] investigated the IBFD amplify-and-forward (AF) system with the direct link using the minimum mean squared error decision feedback equalizer (MMSE-DFE). Their results showed that the outage performance of the IBFD system could be significantly improved if the direct link is appropriately combined with the relaying link at the destination.

In [18], relay selection was proposed for a full-duplex decode-and-forward (FD-DF) relay network with Nakagami- $m$ fading channels. The exact OP expressions were investigated, and their results were shown that the achievable diversity depends on the shape factors of the fading channels and the power scaling scheme of the relay. In [19], the system quality of the DF opportunistic relay network was analyzed in the presence of cochannel interference in the case of cooperative communications. Their results showed that the opportunistic relay selection based on the signal-to-interference-plus-noise ratio (SINR) provides more benefits with an increasing number of relays. In [5], the outage performance of the FD cooperative system quality was analyzed under asymmetric generalized fading conditions. The authors demonstrated that the system performance is much affected by the fading conditions of both the direct and relay links.

Although the outage performance of IBFD relay networks was extensively studied in previous works, the common assumption was that the transceivers were ideal. In practice, most hardware equipment is not ideal and is often influenced by various factors such as manufacturing errors, phase oscillator noises, imbalance in the modulator, nonlinear distortion of the high-power amplifier (HPA) at the transmitter, and the low-noise amplifier (LNA) at the receiver. These hardware impairments degrade the performance and thus should not be neglected in the system design and performance analysis. In fact, the impact of hardware impairments was thoroughly analyzed for the half-duplex relay system in the literature. In [20], the authors analyzed the impact of hardware impairments at both the transmitter and receiver in a dual-hop relay network using AF and DF protocols. In [8], the authors examined the effect of hardware impairments on the system performance of a relay network using switch-and-examine combining with a postselection scheduling scheme in the Rician fading and shadowing channel. Hardware impairments were shown to cause an irreducible outage floor at the high SINR region. The work in [21] investigated and compared the outage performance of the one-way and two-way relay networks under the impact of both hardware impairments and imperfect channel estimation. It was shown in [21] that the one-way relay network has a lower outage floor than the counterpart network. Recently, HI was also considered in the IBFD relay systems [22-27]. In particular, the impact of $\mathrm{HI}$ on the spectral efficiency of a multipair massive MIMO twoway IBFD relay system was analyzed in [22]. The spectral efficiency of a similar system using zero forcing at the relay was evaluated in [23]. A gradient projection-based algorithm to obtain the optimal relay function together with nonalternating and alternating suboptimal solutions was proposed for IBFD AF relay systems with multiple antennas in [24]. The alternating optimization was then extended to design the bidirectional IBFD MIMO OFDM systems with linear precoding and decoding [26]. The performance of the IBFD relay system under the impact of hardware impairments, imperfect channel state information, and imperfect self-interference cancellation was analyzed in [25]. Antenna selection was proposed as a solution to compensate for the performance saturation of the MIMO spatial modulation IBFD relay system [27]. Although the impact of hardware impairments on the performance of the IBFD relay systems was widely analyzed, the case with direct link between the source and the destination nodes was still neglected. Moreover, the exact solution for relay power allocation has not been reported.

Motivated by the previous results, in this paper, we analyze the OP and symbol error probability (SEP) of the IBFD decode-and-forward (DF) relay networks under the effect of the aggregate transceiver impairments and different cooperation scenarios. The contributions of this paper are summarized as follows:

(i) Inspired by the 5G HetNet structure [12], we develop a new system model for a more realistic IBFD relay network with hardware impairments and RSI. Based on this model, performance of the IBFD relay network is analyzed for realistic cooperation situations

(ii) Exact expressions of the signal-to-interferenceplus-noise-and-distortion ratio (SINDR) and outage performance are derived for three cases, i.e., (i) there is no direct link from the source to the destination; (ii) there is a direct link, but the signal from the source is considered interference; and (iii) there is a direct link, and the signal from the source is cooperatively combined with that from the relaying path

(iii) In order to cope with the effect of imperfect selfinterference cancellation (SIC) due to the fullduplex mode, we propose optimal and suboptimal power calculation methods for the relay to minimize the OP. Unlike previous works about a power allocation scheme for the IBFD transmission mode, we obtain the exact optimal transmission power value for the IBFD relay that minimizes the OP 
and SEP of the IBFD relay network. Our results can significantly reduce the computational complexity in the deployment of the IBFD relay system

The remainder of this paper is organized as follows. Section 2 presents the system model of the IBFD relay network. Performance analysis is given in Section 3, followed by the proposed optimal/suboptimal power calculation methods in Section 4. The corresponding numerical results are presented in Section 0. Finally, conclusions are drawn in Section 0. For reading convenience, a summary list of the frequently used mathematical notations is given in Table 1.

\section{System Model}

Let us consider the case of an IBFD DF relay system as shown in Figure 1. In this system, the two terminal nodes $S_{1}$ and $S_{2}$ transmit to the relay node $R$ one at a time while $\mathrm{R}$ can forward the received signal from one terminal node to the other at the same time using the same carrier frequency. Due to the symmetrical communications, we will limit our presentation for the link from $S_{1}$ to $S_{2}$ via $R$. The source node $S_{1}$ and the destination node $S_{2}$ are equipped with a single antenna while the relay $\mathrm{R}$ has two antennas, one for reception and one for transmission. Unlike in the ideal hardware system, the transmitted signal from a relay system with hardware impairment is distorted due to the impact of the transceiver errors such as amplification gain instability, phase noise (PN), high-power amplifier (HPA) nonlinearity, or in-phase/quadrature-phase $(I / Q)$ imbalance. In order to tackle the problem of hardware impairments, various compensation solutions using both analog and digital signal processing have been reported in the literature [21, 28-30]. However, the measurement results demonstrated that the residual impairments after compensation still affect the system as the additive distortion noises which can be modeled by a complex Gaussian variable with zero mean and finite variance $[21,28-30]$. As can be seen from Figure 1, there are two distortion sources in the system, which are denoted by $\eta_{i}(t), i=\{1, \mathrm{R}\}$, where $t$ denotes the time slot. In this paper, we follow the distortion model in [30] which combines the distortion source at the transmitter with that at the receiver. Thus, $\eta_{1}(t)$ denotes the combined distortions at both the transmitter of $S_{1}$ and the receiver of $\mathrm{R}$ and $\eta_{\mathrm{R}}(t)$ for those at both the transmitter of $\mathrm{R}$ and the receiver of $S_{2}$. These combined distortion sources have the following distributions $\eta_{1} \sim C \mathcal{N}\left(0, k_{\mathrm{S}_{1} \mathrm{R}}^{2} P_{1}\right)$ and $\eta_{\mathrm{R}} \sim C \mathcal{N}(0$, $\left.k_{\mathrm{RS}}^{2} P_{\mathrm{R}}\right) ; P_{1}$ and $P_{\mathrm{R}}$ are the average signal power from the source and the relay node, respectively; $k_{\mathrm{S}_{1} \mathrm{R}}^{2}=\left(k_{\mathrm{S}_{1}}^{\mathrm{t}}\right)^{2}+\left(k_{\mathrm{R}}^{\mathrm{r}}\right)^{2}$; $k_{\mathrm{RS}_{2}}^{2}=\left(k_{\mathrm{R}}^{\mathrm{t}}\right)^{2}+\left(k_{\mathrm{S}_{2}}^{\mathrm{r}}\right)^{2}$, where $k_{\mathrm{S}_{1}}^{\mathrm{t}}$ and $k_{\mathrm{R}}^{\mathrm{t}}$ and $k_{\mathrm{R}}^{\mathrm{r}}$ and $k_{\mathrm{S}_{2}}^{\mathrm{r}}$ are, respectively, the level of the impairments at the transmitters of $\mathrm{S}_{1}$ and $\mathrm{R}$ and the receivers of $\mathrm{R}$ and $\mathrm{S}_{2}$. Note that when $k_{\mathrm{S}_{1} \mathrm{R}}=k_{\mathrm{RS}_{2}}=0$, we have an ideal hardware system. The transmit signal from each transmitter can be expressed as $s_{i}(t)+\eta_{i}(t)$. We assume that the processing delay at the relay $\mathrm{R}$ equals one symbol period. Therefore, a symbol
TABLE 1: List of the frequently used mathematical notations.

\begin{tabular}{lc}
\hline Notation & Description \\
\hline$P_{1}$ & The average transmit power at the source node $\mathrm{S}_{1}$ \\
$P_{\mathrm{R}}$ & The average transmit power at the relay node $\mathrm{R}$ \\
$\operatorname{Prob}\{\mathscr{A}\}$ & The probability of an event $\mathscr{A}$ \\
OP & Outage probability \\
$\mathrm{OP}^{(\ell)_{\text {ex }}}$ & Exact OP of the case $\ell$ with hardware impairments \\
$\mathrm{OP}^{(\ell) \_ \text {ex } \_ \text {id }}$ & Exact OP of the case $\ell$ with ideal hardware \\
$\mathrm{OP}^{(\ell) \_ \text {ap }}$ & Approximate OP of the case $\ell$ with hardware \\
$F_{\rho}()$. & impairments \\
$f_{\rho}()$. & Cumulative distribution function (CDF) \\
$\mathscr{C} \mathscr{N}\left(\mu, \sigma^{2}\right)$ & Complex Gaussian distribution with mean $\mu$ and \\
$\mathbb{E}\{\cdot\}$ & variance of $\sigma^{2}$ \\
$\rho=|h|^{2}$ & The expectation operator \\
$\Omega=\mathbb{E}\{\rho\}$ & The instantaneous channel gain \\
$\mathrm{SEP}$ & Symbol error probability
\end{tabular}

which was received by $\mathrm{R}$ at time slot $t-1$ will be transmitted at time slot $t$. It means that $s_{\mathrm{R}}(t)=s_{1}(t-1)$ if $\mathrm{R}$ decodes successfully the received signal. The received signal at the relay and the destination is then given, respectively, by

$$
\begin{aligned}
& y_{\mathrm{R}}(t)=h_{1 \mathrm{R}} \tilde{s}_{1}(t)+\tilde{h}_{\mathrm{RR}} \tilde{s}_{\mathrm{R}}(t)+z_{\mathrm{R}}(t), \\
& y_{2}(t)=h_{\mathrm{R} 2} \tilde{s}_{\mathrm{R}}(t)+h_{12} \tilde{s}_{1}(t)+z_{2}(t),
\end{aligned}
$$

where $\tilde{s}_{1}(t)$ and $\tilde{s}_{\mathrm{R}}(t)$, respectively, denote the transmitted signals from the source and the relay with $\tilde{s}_{1}(t) \triangleq s_{1}(t)+$ $\eta_{1}(t)$ and $\tilde{s}_{\mathrm{R}}(t) \triangleq s_{\mathrm{R}}(t)+\eta_{\mathrm{R}}(t) ; \eta_{1}(t)$ and $\eta_{\mathrm{R}}(t)$ denote the effect of hardware impairments at the transceivers with $\eta_{1} \sim C \mathcal{N}\left(0, k_{\mathrm{S}_{1} \mathrm{R}}^{2} P_{1}\right)$ and $\eta_{\mathrm{R}} \sim C \mathcal{N}\left(0, k_{\mathrm{RS}}^{2} P_{\mathrm{R}}\right) ; h_{1 \mathrm{R}}, h_{\mathrm{R} 2}$, and $h_{12}$ are the fading coefficients of the links from $S_{1}$ to $R$, from $\mathrm{R}$ to $\mathrm{S}_{2}$, and from $\mathrm{S}_{1}$ to $\mathrm{S}_{2}$, respectively; $\tilde{h}_{\mathrm{RR}}$ is the RSI channel from the transmitting antenna to the receiving antenna at the FD relay $\mathrm{R}$ after self-interference cancellation; and $z_{\mathrm{R}}(t)$ and $z_{2}(t)$ denote the AWGN with zero mean and variance $\sigma_{\mathrm{R}}^{2}$ and $\sigma_{2}^{2}$, respectively, $z_{\mathrm{R}} \sim C \mathcal{N}$ $\left(0, \sigma_{\mathrm{R}}^{2}\right)$ and $z_{2} \sim C \cdot \mathcal{N}\left(0, \sigma_{2}^{2}\right)$.

Note from (1) that the term $\tilde{h}_{\mathrm{RR}} \tilde{s}_{\mathrm{R}}(t)$ represents the SI at $\mathrm{R}$ and its power is significantly larger than that of the intended signal due to small distance between transmitter and receiver antennas of $\mathrm{R}$. In order for the relay to decode the intended signal successfully, three popular SIC techniques can be applied to make the SI to the noise level, including those in the propagation, analog and digital domains [4, 31]. The RSI after these SIC techniques can be modeled by a Gaussian random variable, denoted 


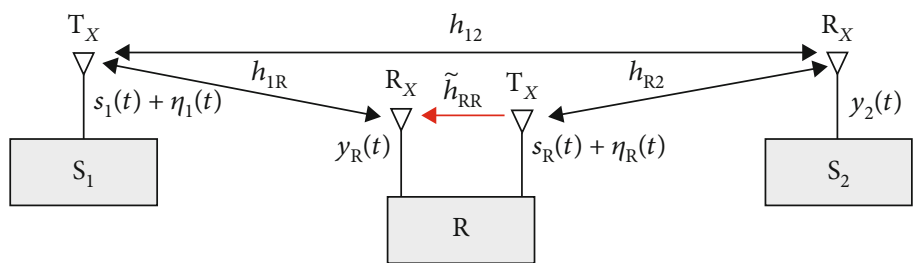

FIGURE 1: Block diagram of the IBFD relay system with transceiver impairments.

by $I_{\mathrm{R}}$ with zero mean and variance of $\sigma_{\mathrm{RSI}}^{2}$, i.e., $I_{\mathrm{R}} \sim C \mathcal{N}(0$, $\left.\sigma_{\mathrm{RSI}}^{2}\right)[4,31]$. The received signal at $\mathrm{R}$ after $\mathrm{SIC}$ can be now expressed as

$$
y_{\mathrm{R}}(t)=h_{1 \mathrm{R}} \tilde{s}_{1}(t)+I_{\mathrm{R}}(t)+z_{\mathrm{R}}(t) .
$$

In practice, due to the distance variation and shadowing, the transmitted signal from the source can reach the destination in some cases. Therefore, the system quality in realistic conditions varies according to the following three cases: (1) there is no direct link from $S_{1}$ to $S_{2}$; (2) there is a direct link from $S_{1}$ to $S_{2}$, but its signal is considered interference at $S_{2}$; and (3) there is a direct link from $S_{1}$ to $S_{2}$, and its signal is combined with that of the relaying link using cooperative communication.

Note that when the DF relaying protocol is used, the endto-end SINDR of the system is the minimum of the SINDRs of the links from the source to the relay and from the relay to the destination. Thus, the end-to-end SINDR of the DF relay system is given by

$$
\gamma=\min \left\{\gamma_{\mathrm{R}}, \gamma_{\mathrm{D}}\right\}
$$

where $\gamma_{\mathrm{R}}$ and $\gamma_{\mathrm{D}}$ are the SINDRs at the relay and the destination, respectively.

2.1. Case 1: Without the Direct Link. In this case, the transmitted signal from the source $S_{1}$ does not reach the destination $\mathrm{S}_{2}$ due to long distance. The destination receives only the relayed signal from R. Using (3) and (2), we can obtain the SINDRs at the relay $\mathrm{R}$ and the destination $S_{2}$ for Case 1, respectively, as

$$
\gamma_{\mathrm{R}}^{(1)}=\frac{\left|h_{1 \mathrm{R}}\right|^{2} P_{1}}{\left|h_{1 \mathrm{R}}\right|^{2} k_{\mathrm{S}_{1} \mathrm{R}}^{2} P_{1}+\tilde{\Omega}_{\mathrm{R}} P_{\mathrm{R}}+\sigma_{\mathrm{R}}^{2}}=\frac{\rho_{1} P_{1}}{\rho_{1} k_{\mathrm{S}_{1} \mathrm{R}}^{2} P_{1}+\sigma_{\mathrm{RSI}}^{2}+\sigma_{\mathrm{R}}^{2}},
$$

$$
\gamma_{\mathrm{D}}^{(1)}=\frac{\left|h_{\mathrm{R} 2}\right|^{2} P_{\mathrm{R}}}{\left|h_{\mathrm{R} 2}\right|^{2} k_{\mathrm{R} S_{2}}^{2} P_{\mathrm{R}}+\sigma_{2}^{2}}=\frac{\rho_{2} P_{\mathrm{R}}}{\rho_{2} k_{\mathrm{RS}}^{2} P_{\mathrm{R}}+\sigma_{2}^{2}},
$$

where $\rho_{1}=\left|h_{1 \mathrm{R}}\right|^{2}$ and $\rho_{2}=\left|h_{\mathrm{R} 2}\right|^{2}$ are the channel gains of the links from $S_{1}$ to $R$ and from $R$ to $S_{2}$, respectively; $\sigma_{\mathrm{RSI}}^{2}$ denotes the RSI at the relay after SIC. It is noted that, after SIC, the RSI can be modeled by a complex Gaussian distributed random variable $[2-6,9,10,32,33]$ with zero mean and variance $\sigma_{\mathrm{RSI}}^{2}=\tilde{\Omega}_{\mathrm{R}} P_{\mathrm{R}}$, where $\tilde{\Omega}_{\mathrm{R}}$ is defined as the SIC capability of the relay node.

2.2. Case 2: With the Direct Link but No Cooperative Communication. In this case, the destination receives the transmitted signal from the source via both the relaying and direct links. However, due to time misalignment, the signal via the direct link is considered interference. Therefore, the SINDRs at the relay and the destination are, respectively, given by

$$
\begin{aligned}
\gamma_{\mathrm{R}}^{(2)} & =\gamma_{\mathrm{R}}^{(1)}=\frac{\rho_{1} P_{1}}{\rho_{1} k_{\mathrm{S}_{1} \mathrm{R}}^{2} P_{1}+\sigma_{\mathrm{RS} I}^{2}+\sigma_{\mathrm{R}}^{2}}, \\
\gamma_{\mathrm{D}}^{(2)} & =\frac{\left|h_{\mathrm{R} 2}\right|^{2} P_{\mathrm{R}}}{\left|h_{\mathrm{R} 2}\right|^{2} k_{\mathrm{RS}}^{2} P_{\mathrm{R}}+\left|h_{12}\right|^{2} P_{1}\left(1+k_{\mathrm{S}_{1} \mathrm{R}}^{2}\right)+\sigma_{2}^{2}} \\
& =\frac{\rho_{2} P_{\mathrm{R}}}{\rho_{2} k_{\mathrm{RS}}^{2} P_{\mathrm{R}}+\rho_{3} P_{1}\left(1+k_{\mathrm{S}_{1} \mathrm{R}}^{2}\right)+\sigma_{2}^{2}},
\end{aligned}
$$

where $\rho_{3}=\left|h_{12}\right|^{2}$ is the channel gain of the link from $S_{1}$ to $S_{2}$.

2.3. Case 3: With the Direct Link and Cooperative Communication. In this case, the signal from the source via the relaying link and that via the direct link are aligned (the system is thus delay limited) and combined in order to maximize the SINDR at the destination and remove the intersymbol interference (ISI) due to the processing delay at the relay node. For such purpose, we adopt the MMSE-DFE proposed in [17] at the destination. In order to estimate the transmitted symbol, the equalizer needs to be trained and operates in an adaptive fashion. This results in training delay and requires that the channel be slowly varying. Moreover, since the transmitted signals from the source and relay nodes also contain HI distortions $\eta_{1}(t)$ and $\eta_{\mathrm{R}}(t)$, these components are considered additive noises by the MMSE-DFE. The SINDRs at the relay and the destination are then given, respectively, by

$$
\gamma_{\mathrm{R}}^{(3)}=\gamma_{\mathrm{R}}^{(1)}=\frac{\rho_{1} P_{1}}{\rho_{1} k_{\mathrm{S}_{1} \mathrm{R}}^{2} P_{1}+\sigma_{\mathrm{RSI}}^{2}+\sigma_{\mathrm{R}}^{2}},
$$




$$
\begin{aligned}
\gamma_{\mathrm{D}}^{(3)} & =\frac{\left|h_{\mathrm{R} 2}\right|^{2} P_{\mathrm{R}}+\left|h_{12}\right|^{2} P_{1}}{\left|h_{\mathrm{R} 2}\right|^{2} k_{\mathrm{RS}}^{2} P_{\mathrm{R}}+\left|h_{12}\right|^{2} k_{\mathrm{S}_{1} \mathrm{R}}^{2} P_{1}+\sigma_{2}^{2}} \\
& =\frac{\rho_{2} P_{\mathrm{R}}+\rho_{3} P_{1}}{\rho_{2} k_{\mathrm{RS}_{2}}^{2} P_{\mathrm{R}}+\rho_{3} k_{\mathrm{S}_{1} \mathrm{R}}^{2} P_{1}+\sigma_{2}^{2}} .
\end{aligned}
$$

\section{System Performance}

3.1. Outage Probability. The outage probability is the probability of a failure in any communication link between $S_{1}$, $R$, and $S_{2}$. An outage event occurs when the mutual information is lower than the minimum required data rate $\mathscr{R}_{j},(j=1,2)$, i.e.,

$$
\log _{2}\left(1+\gamma_{j}\right)<\mathscr{R}_{j}
$$

where $\gamma_{j}$ and $\mathscr{R}_{j}$ are the SINDR and the minimum required data rate of link $j$, respectively. Let $\mathscr{R}_{1}$ be the minimum required data rate from $\mathrm{S}_{1}$ to $\mathrm{R}$ and $\mathscr{R}_{2}$ be that from $\mathrm{R}$ to $\mathrm{S}_{2}$. Also, for simplicity, let $\mathscr{R}_{1}=\mathscr{R}_{2}=\mathscr{R}$. As a result, an outage event occurs when

$$
\begin{array}{r}
\log _{2}\left(1+\gamma_{\mathrm{R}}\right)<\mathscr{R} \\
\text { or } \log _{2}\left(1+\gamma_{\mathrm{D}}\right)<\mathscr{R},
\end{array}
$$

which is equivalent to

$$
\begin{aligned}
& \gamma_{\mathrm{R}}<2^{\mathscr{R}}-1 \\
& \text { or } \gamma_{\mathrm{D}}<2^{\mathscr{R}}-1 .
\end{aligned}
$$

The outage probability is then defined as $\mathrm{OP}=$ $\operatorname{Prob}\left(\gamma<2^{\mathscr{R}}-1\right)$. Let $x=2^{\mathscr{R}}-1$, and using the rule of addition $\operatorname{Prob}\{\mathscr{A} \cup \mathscr{B}\}=\operatorname{Prob}\{\mathscr{A}\}+\operatorname{Prob}\{\mathscr{B}\}-\operatorname{Prob}\{\mathscr{A}\}$ $\operatorname{Prob}\{\mathscr{B}\}$ for independent events $\mathscr{A}$ and $\mathscr{B}$, we can obtain the OP as follows:

$$
\begin{aligned}
\mathrm{OP} & =\operatorname{Prob}\left\{\left(\gamma_{\mathrm{R}}<x\right) \cup\left(\gamma_{\mathrm{D}}<x\right)\right\}=\operatorname{Prob}\left\{\min \left[\gamma_{\mathrm{R}}, \gamma_{\mathrm{D}}\right]<x\right\} \\
& =\mathrm{OP}_{\mathrm{R}}+\mathrm{OP}_{\mathrm{D}}-\mathrm{OP}_{\mathrm{R}} \mathrm{OP}_{\mathrm{D}}
\end{aligned}
$$

where $\mathrm{OP}_{\mathrm{R}}=\operatorname{Prob}\left\{\gamma_{\mathrm{R}}<x\right\}$ and $\mathrm{OP}_{\mathrm{D}}=\operatorname{Prob}\left\{\gamma_{\mathrm{D}}<x\right\}$.

Under the Rayleigh fading channel, the cumulative distribution function (CDF) $F_{\rho}($.$) and probability density func-$ tion (PDF) $f_{\rho}($.$) of the channel gains \left(\rho_{l}=\left|h_{l}\right|^{2}, l=1,2,3\right)$ are given by

$$
\begin{aligned}
& F_{\rho_{l}}(x)=1-e^{-\left(x / \Omega_{l}\right)}, \quad x \geqslant 0, \\
& f_{\rho_{l}}(x)=\frac{1}{\Omega_{l}} e^{-\left(x / \Omega_{l}\right)}, \quad x \geqslant 0,
\end{aligned}
$$

where $\Omega_{l}=\mathbb{E}\left\{\left|h_{l}\right|^{2}\right\}$.

Under the effect of both Rayleigh fading and hardware impairments, using the above CDF and PDF of the channel gains, we can get the OPs of the system for each cooperation situation in the following parts.

\subsubsection{Exact $O P$}

(1) Case 1: Without the Direct Link. Denoting $\mathrm{OP}_{\mathrm{R}}^{(1) \text { ex }}=$ $\operatorname{Prob}\left\{\gamma_{\mathrm{R}}^{(1)}<x\right\}$ and $\mathrm{OP}_{\mathrm{D}}^{(1) \_ \text {ex }}=\operatorname{Prob}\left\{\gamma_{\mathrm{D}}^{(1)}<x\right\}$, we have

$$
\begin{aligned}
& \mathrm{OP}_{\mathrm{R}}^{(1) \_\mathrm{ex}}=\operatorname{Prob}\left\{\gamma_{\mathrm{R}}^{(1)}<x\right\} \\
& = \begin{cases}F_{\rho_{1}}\left(\frac{\left(\sigma_{\mathrm{R}}^{2}+\sigma_{\mathrm{RSI}}^{2}\right) x}{P_{1}\left(1-k_{\mathrm{S}_{1} \mathrm{R}}^{2} x\right)}\right), & x<\frac{1}{k_{\mathrm{S}_{1} \mathrm{R}}^{2}}, \\
1, & x \geqslant \frac{1}{k_{\mathrm{S}_{1} \mathrm{R}}^{2}},\end{cases} \\
& = \begin{cases}1-e^{-\alpha_{1}(x)}, & x<\frac{1}{k_{\mathrm{S}_{1} \mathrm{R}}^{2}}, \\
1, & x \geqslant \frac{1}{k_{\mathrm{S}_{1} \mathrm{R}}^{2}} .\end{cases}
\end{aligned}
$$

Similarly,

$$
\mathrm{OP}_{\mathrm{D}}^{(1) \_\mathrm{ex}}=\operatorname{Prob}\left\{\gamma_{\mathrm{D}}^{(1)}<x\right\}= \begin{cases}1-e^{-\alpha_{2}(x)}, & x<\frac{1}{k_{\mathrm{RS}}^{2}}, \\ 1, & x \geqslant \frac{1}{k_{\mathrm{RS}}^{2}} .\end{cases}
$$

Using (14), the OP of the system becomes

$$
\mathrm{OP}^{(1) \_\mathrm{ex}}= \begin{cases}1-e^{-\alpha_{1}(x)-\alpha_{2}(x)}, & x<d, \\ 1, & x \geqslant d,\end{cases}
$$

where $\alpha_{1}(x)=\left(\sigma_{\mathrm{R}}^{2}+\sigma_{\mathrm{RSI}}^{2}\right) x / \Omega_{1} P_{1}\left(1-k_{\mathrm{S}_{1} \mathrm{R}}^{2} x\right), \alpha_{2}(x)=\sigma_{2}^{2} x / \Omega_{2}$ $P_{\mathrm{R}}\left(1-k_{\mathrm{RS}_{2}}^{2} x\right)$, and $d=\min \left(1 / k_{\mathrm{S}_{1} \mathrm{R}}^{2}, 1 / k_{\mathrm{RS}_{2}}^{2}\right)$. 

by

Note that for the case of ideal hardware, the OP is given

$$
\mathrm{OP}^{(1) \_\mathrm{ex} \_\mathrm{id}}=1-e^{-\alpha_{1}^{\mathrm{id}}(x)-\alpha_{2}^{\mathrm{id}}(x)},
$$

where $\alpha_{1}^{\mathrm{id}}(x)=\left(\sigma_{\mathrm{R}}^{2}+\sigma_{\mathrm{RSI}}^{2}\right) x / \Omega_{1} P_{1}$ and $\alpha_{2}^{\mathrm{id}}(x)=\sigma_{2}^{2} x / \Omega_{2} P_{\mathrm{R}}$.

(2) Case 2: With the Direct Link but No Cooperative Communication. Since $\gamma_{\mathrm{R}}^{(1)}=\gamma_{\mathrm{R}}^{(2)}=\gamma_{\mathrm{R}}^{(3)}$, we have

$$
\mathrm{OP}_{\mathrm{R}}^{(1){ }^{\mathrm{ex}}}=\mathrm{OP}_{\mathrm{R}}^{(2) \_\mathrm{ex}}=\mathrm{OP}_{\mathrm{R}}^{(3) \_\mathrm{ex}} \text {. }
$$

In order to calculate $\mathrm{OP}_{\mathrm{D}_{-}}^{(2) \text { ex }}$ using (8), we have

$$
\begin{aligned}
F_{\gamma_{\mathrm{D}}^{(2)}}(x) & =\operatorname{Prob}\left\{\gamma_{\mathrm{D}}^{(2)}<x\right\}=\operatorname{Prob}\left\{\rho_{2} P_{\mathrm{R}}\left(1-k_{\mathrm{RS}_{2}}^{2} x\right)\right. \\
& \left.<\left[\rho_{3} P_{1}\left(1+k_{\mathrm{S}_{1} \mathrm{R}}^{2}\right)+\sigma_{2}^{2}\right] x\right\} .
\end{aligned}
$$

This is the joint CDF of two independent random variables $\rho_{2}$ and $\rho_{3}$. First, consider the case $1-k_{\mathrm{RS}_{2}}^{2} x \leq 0$, which means $x \geq 1 / k_{\mathrm{RS}}^{2}$. In this case, the inequality $\rho_{2} P_{\mathrm{R}}(1-$ $\left.k_{\mathrm{RS}_{2}}^{2} x\right)<\left[\rho_{3} P_{1}\left(1+k_{\mathrm{S}_{1} \mathrm{R}}^{2}\right)+\sigma_{2}^{2}\right] x$ is always true due to the fact that $\rho_{2} P_{\mathrm{R}}\left(1-k_{\mathrm{RS}_{2}}^{2} x\right) \leq 0$ and $\left(\rho_{3} P_{1}\left(1+k_{\mathrm{S}_{1} \mathrm{R}}^{2}\right)+\sigma_{2}^{2}\right) x>0$. Therefore, $F_{\gamma_{\mathrm{D}}^{(2)}}(x)=1$. Next, we consider the case $1-k_{\mathrm{RS}_{2}}^{2} x>$ 0 , which means $\rho_{2} P_{\mathrm{R}}\left(1-k_{\mathrm{RS}}^{2} x\right)>0$. Since $\rho_{2}$ and $\rho_{3}$ are the instantaneous channel gains, we have $\rho_{2}, \rho_{3} \in(0, \infty)$. To derive the $\mathrm{CDF}$ in this case, we change the above probability to a new equivalent one which has only one random variable. Using the property of a function of two random variables in Section 5.8 of [34], we can convert the joint CDF of these two independent variables into a function with double integral in which one variable is considered a constant while calculating the other. Then, by applying the result in Section 5.7 of [34], we can obtain

$$
\begin{aligned}
F_{\gamma_{\mathrm{D}}^{(2)}}(x) & =\int_{0}^{\infty} \operatorname{Prob}\left\{\rho_{2}<\frac{\left[\rho_{3} P_{1}\left(1+k_{\mathrm{S}_{1} \mathrm{R}}^{2}\right)+\sigma_{2}^{2}\right] x}{P_{\mathrm{R}}\left(1-k_{\mathrm{RS}}^{2} x\right)} \mid \rho_{3}\right\} f_{\rho_{3}}\left(\rho_{3}\right) d \rho_{3} \\
& =\int_{0}^{\infty} F_{\rho_{2}}\left\{\frac{\left[\rho_{3} P_{1}\left(1+k_{\mathrm{S}_{1} \mathrm{R}}^{2}\right)+\sigma_{2}^{2}\right] x}{P_{\mathrm{R}}\left(1-k_{\mathrm{RS}}^{2} x\right)}\right\} f_{\rho_{3}}\left(\rho_{3}\right) d \rho_{3} .
\end{aligned}
$$

In summary, we have $\mathrm{OP}_{\mathrm{D}^{-}}^{(2)}{ }^{\mathrm{ex}}$ given by

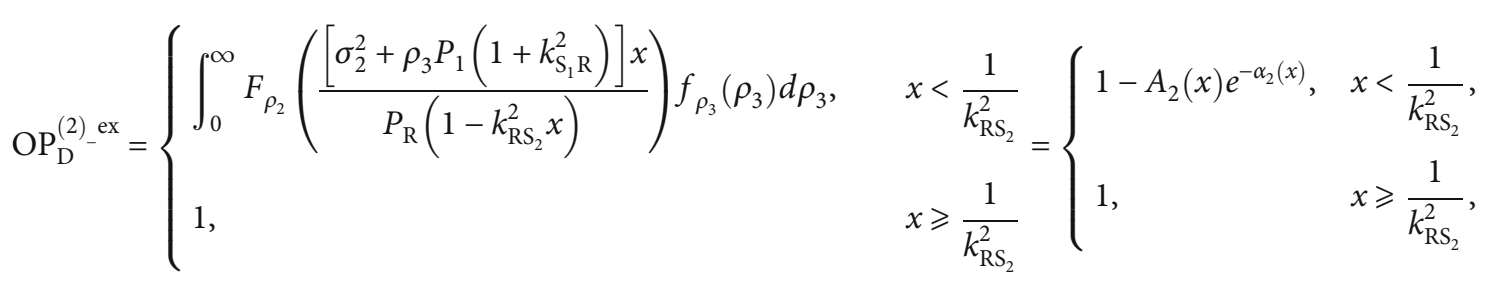

where $A_{2}(x)=\Omega_{2} P_{\mathrm{R}}\left(1-k_{\mathrm{RS}_{2}}^{2} x\right) /\left(\Omega_{2} P_{\mathrm{R}}\left(1-k_{\mathrm{RS}}^{2} x\right)+\Omega_{3} P_{1}(1\right.$ $\left.\left.+k_{\mathrm{S}_{1} \mathrm{R}}^{2}\right) x\right)$.

Thus, OP of the system for this case is then given by

$$
\mathrm{OP}^{(2) \_\mathrm{ex}}= \begin{cases}1-A_{2}(x) e^{-\alpha_{1}(x)-\alpha_{2}(x)}, & x<d, \\ 1, & x \geqslant d .\end{cases}
$$

In the case of ideal hardware, the system OP is given by

$$
\mathrm{OP}^{(2) \_ \text {ex_id }}(x)=1-A_{2}^{\mathrm{id}}(x) e^{-\alpha_{1}^{\mathrm{id}}(x)-\alpha_{2}^{\mathrm{id}}(x)}
$$

where $A_{2}^{\mathrm{id}}(x)=\Omega_{2} P_{\mathrm{R}} /\left(\Omega_{2} P_{\mathrm{R}}+\Omega_{3} P_{1} x\right)$.

(3) Case 3: With the Direct Link and Cooperative Communication. In this case, using the same derivation for $\mathrm{OP}_{\mathrm{D}}^{(2)}{ }_{-}^{\mathrm{ex}}$, we can have $\mathrm{OP}_{\mathrm{D}}^{(3)}{ }^{\text {ex }}$ as follows:

$$
\begin{aligned}
& \mathrm{OP}_{\mathrm{D}}^{(3) \_\mathrm{ex}}=\operatorname{Prob}\left\{\gamma_{\mathrm{D}}^{(3)}<x\right\} \\
& =\operatorname{Prob}\left\{\rho_{2} P_{\mathrm{R}}\left(1-k_{\mathrm{RS}_{2}}^{2} x\right)+\rho_{3} P_{1}\left(1-k_{\mathrm{S}_{1} \mathrm{R}}^{2} x\right)<\sigma_{2}^{2} x\right\} \\
& = \begin{cases}1-A_{3}(x) e^{-\alpha_{2}(x)}-B_{3}(x) e^{-\alpha_{3}(x)}, & x<d, G(x) \neq 0, \\
1-\left(1+\alpha_{2}(x)\right) e^{-\alpha_{2}(x)}, & x<d, G(x)=0, \\
1-A_{3}(x) e^{-\alpha_{2}(x)}, & \frac{1}{k_{\mathrm{S}_{1} \mathrm{R}}^{2}} \leqslant x<\frac{1}{k_{\mathrm{RS}_{2}}^{2}}, \\
1-B_{3}(x) e^{-\alpha_{3}(x)}, & \frac{1}{k_{\mathrm{RS}_{2}}^{2}} \leqslant x<\frac{1}{k_{\mathrm{S}_{1} \mathrm{R}}^{2}}, \\
1, & x \geqslant d^{\prime},\end{cases}
\end{aligned}
$$

where $A_{3}(x)=\Omega_{2} P_{\mathrm{R}}\left(1-k_{\mathrm{RS}_{2}}^{2} x\right) /\left(\Omega_{2} P_{\mathrm{R}}\left(1-k_{\mathrm{RS}}^{2} x\right)-\Omega_{3} P_{1}(1\right.$ $\left.\left.-k_{\mathrm{S}_{1} \mathrm{R}}^{2} x\right)\right), \quad B_{3}(x)=\Omega_{3} P_{1}\left(1-k_{\mathrm{S}_{1} \mathrm{R}}^{2} x\right) /\left(\Omega_{3} P_{1}\left(1-k_{\mathrm{S}_{1} \mathrm{R}}^{2} x\right)-\Omega_{2}\right.$ $\left.P_{\mathrm{R}}\left(1-k_{\mathrm{RS}_{2}}^{2} x\right)\right), d^{\prime}=\max \left(1 / k_{\mathrm{S}_{1} \mathrm{R}}^{2}, 1 / k_{\mathrm{RS}_{2}}^{2}\right), \quad \alpha_{3}(x)=\sigma_{2}^{2} x / \Omega_{3} P_{1}$ $\left(1-k_{\mathrm{S}_{1} \mathrm{R}}^{2} x\right)$, and $G(x)=\Omega_{3} P_{1}\left(1-k_{\mathrm{S}_{1} \mathrm{R}}^{2} x\right)-\Omega_{2} P_{\mathrm{R}}\left(1-k_{\mathrm{RS}_{2}}^{2} x\right)$. 
Combining $\mathrm{OP}_{\mathrm{D}}^{(3) \_\mathrm{ex}}$ in (26) with $\mathrm{OP}_{\mathrm{R}}^{(3) \_\mathrm{ex}}$ in (20), we have the $\mathrm{OP}$ for the case of cooperative communication $\mathrm{OP}^{(3) \_\mathrm{ex}}$ as follows:

$\mathrm{OP}^{(3) \_\mathrm{ex}}= \begin{cases}1-A_{3}(x) e^{-\alpha_{1}(x)-\alpha_{2}(x)}-B_{3}(x) e^{-\alpha_{1}(x)-\alpha_{3}(x)}, & x<d, G(x) \neq 0, \\ 1-\left(1+\alpha_{2}(x)\right) e^{-\alpha_{1}(x)-\alpha_{2}(x),} & x<d, G(x)=0, \\ 1-B_{3}(x) e^{-\alpha_{1}(x)-\alpha_{3}(x)}, & \frac{1}{k_{\mathrm{RS}}^{2}} \leqslant x<\frac{1}{k_{\mathrm{S}_{1} \mathrm{R}}^{2}}, \\ 1, & x \geqslant \frac{1}{k_{\mathrm{S}_{1} \mathrm{R}}^{2}} .\end{cases}$

In the case of ideal hardware, we have

$\mathrm{OP}^{(3) \_ \text {ex_id }}= \begin{cases}1-A_{3}^{\mathrm{id}} e^{-\alpha_{1}^{\mathrm{id}}(x)-\alpha_{2}^{\mathrm{id}}(x)}-B_{3}^{\mathrm{id}} e^{-\alpha_{1}^{\mathrm{id}}(x)-\alpha_{3}^{\mathrm{id}}(x)}, & G \neq 0, \\ 1-\left(1+\alpha_{2}^{\mathrm{id}}(x)\right) e^{-\alpha_{1}^{\mathrm{id}}(x)-\alpha_{2}^{\mathrm{id}}(x)}, & G=0,\end{cases}$

where $A_{3}^{\text {id }}=\Omega_{2} P_{\mathrm{R}} /\left(\Omega_{2} P_{\mathrm{R}}-\Omega_{3} P_{1}\right), \quad B_{3}^{\text {id }}=\Omega_{3} P_{1} /\left(\Omega_{3} P_{1}-\Omega_{2}\right.$ $\left.P_{\mathrm{R}}\right), \alpha_{3}^{\text {id }}(x)=\sigma_{2}^{2} x / \Omega_{3} P_{1}$, and $G=\Omega_{3} P_{1}-\Omega_{2} P_{\mathrm{R}}$.

3.1.2. Asymptotic OP. In order to gain a better insight into the effect of hardware impairments on the system performance of the IBFD relay network, we derive the asymptotic OP using the assumption that the transmit power is extremely large. Using the Taylor series expansion, $e^{x} \approx 1+x$ when $x \longrightarrow 0$ and let the transmit power increase to infinity, i.e., $P_{j} \longrightarrow \infty, j=1, \mathrm{R}$, and we assume that $P_{1}=P_{\mathrm{R}}$. Since $\lim _{P_{j} \rightarrow \infty} \alpha_{i}(x)=0, i=1,2,3$, we have

$\lim _{P_{j} \rightarrow \infty} \mathrm{OP}^{(1) \_\mathrm{ex}}= \begin{cases}0, & x<d, \\ 1, & x \geqslant d,\end{cases}$

$\lim _{P_{j} \rightarrow \infty} \mathrm{OP}^{(2) \_\mathrm{ex}}= \begin{cases}1-\frac{\Omega_{2}\left(1-k_{\mathrm{RS}_{2}}^{2} x\right)}{\Omega_{2}\left(1-k_{\mathrm{RS}}^{2} x\right)+\Omega_{3}\left(1+k_{\mathrm{S}_{1} \mathrm{R}}^{2}\right) x}, & x<d, \\ 1, & x \geqslant d,\end{cases}$

$$
\lim _{P_{j} \rightarrow \infty} \mathrm{OP}^{(3) \_\mathrm{ex}}= \begin{cases}1-A_{3}^{\infty}(x)-B_{3}^{\infty}(x), & x<d, G(x) \neq 0, \\ 0, & x<d, G(x)=0, \\ 1-B_{3}^{\infty}(x), & \frac{1}{k_{\mathrm{RS}_{2}}^{2}} \leqslant x<\frac{1}{k_{\mathrm{S}_{1} \mathrm{R}}^{2}}, \\ 1, & x \geqslant \frac{1}{k_{\mathrm{S}_{1} \mathrm{R}}^{2}},\end{cases}
$$

where $A_{3}^{\infty}(x)=\Omega_{2}\left(1-k_{R}^{2} x\right) /\left(\Omega_{2}\left(1-k_{\mathrm{RS}}^{2} x\right)-\Omega_{3}\left(1-k_{\mathrm{S}_{1} \mathrm{R}}^{2} x\right)\right)$ and $B_{3}^{\infty}(x)=\Omega_{3}\left(1-k_{\mathrm{S}_{1} \mathrm{R}}^{2} x\right) / \Omega_{3}\left(1-k_{\mathrm{S}_{1} \mathrm{R}}^{2} x\right)-\Omega_{2}\left(1-k_{\mathrm{RS}_{2}}^{2} x\right)$.
On the other hand, we have

$\mathrm{OP}^{(1) \_\mathrm{ap}} \approx \begin{cases}\alpha_{1}(x)+\alpha_{2}(x), & x<d, \\ 1, & x \geqslant d,\end{cases}$
$\mathrm{OP}^{(2) \_\mathrm{ap}} \approx \begin{cases}1-A_{2}(x)\left[1-\alpha_{1}(x)-\alpha_{2}(x)\right], & x<d, \\ 1, & x \geqslant d,\end{cases}$

$\mathrm{OP}^{(3) \_a \mathrm{p}} \approx \begin{cases}\alpha_{1}(x)+A_{3}(x) \alpha_{2}(x)+B_{3}(x) \alpha_{3}(x), & x<d, G(x) \neq 0, \\ 1-\left(1+\alpha_{2}(x)\right)\left[1-\alpha_{1}(x)-\alpha_{2}(x)\right], & x<d, G(x)=0, \\ 1-B_{3}(x)\left[1-\alpha_{1}(x)-\alpha_{3}(x)\right], & \frac{1}{k_{\mathrm{RS}}^{2}} \leqslant x<\frac{1}{k_{\mathrm{S}_{1} \mathrm{R}}^{2}}, \\ 1, & x \geqslant \frac{1}{k_{\mathrm{S}_{1} \mathrm{R}}^{2}} .\end{cases}$

From (29), (30), and (31), we can see clearly that hardware impairments cause the OP to have an irreducible error floor. Thus, careful design must be taken during the process of manufacturing transceivers in order to avoid this outage floor.

3.2. System Throughput. The throughput of the IBFD DF relay system can be determined from the OP as follows:

$$
\mathscr{T}(x)=\mathscr{R}(1-\mathrm{OP}),
$$

where $\mathscr{R}$ is the given transmission rate (bit/s/Hz) and OP is the outage probability of the system, which is given in (18), (24), and (27) for the three cases of cooperation.

3.3. Symbol Error Probability. For most common modulation schemes, the SEP of the system is given by [35]

$$
\begin{aligned}
\operatorname{SEP}_{\ell} & =\alpha \mathbb{E}\left\{Q\left(\sqrt{\beta \gamma_{\ell}}\right)\right\} \\
& =\frac{\alpha}{\sqrt{2 \pi}} \int_{0}^{\infty} F_{\ell}\left(\frac{t^{2}}{\beta}\right) e^{-\left(t^{2} / 2\right)} d t, \quad \ell=1,2,3,
\end{aligned}
$$

where $Q(x)=1 / \sqrt{2 \pi} \int_{x}^{\infty} e^{-t^{2} / 2} d t$ is the Gaussian function, $\gamma_{\ell}$ is the received SINDR obtained from (5), (6), (7), (8), (9), and (10), and $\alpha$ and $\beta$ are decided by the employed modulation schemes, e.g., $\alpha=\beta=1$ for the orthogonal binary frequency-shift keying (BFSK) modulation and $\alpha=1, \beta=2$ for the binary phase-shift keying (BPSK) modulation [35]. $F_{\ell}(x)$ are the cumulative distribution functions (CDFs) of SINDR. Replace $x=t^{2} / \beta$, and the $\mathrm{SEP}_{\ell}$ for each cooperation situation is calculated as

$$
\operatorname{SEP}_{\ell}=\frac{\alpha \sqrt{\beta}}{2 \sqrt{2 \pi}} \int_{0}^{\infty} \frac{e^{-\beta x / 2}}{\sqrt{x}} F_{\ell}(x) d x
$$


Using the definition of outage probability, we can replace CDFs $F_{\ell}(x)$ by OPs given by (18), (24), and (27). Since O $\mathrm{P}^{(\ell) \_e x}$ is complicated, it is not possible to obtain the closed form or approximation for (37) even using Mathematica software. However, it is worth noting that since $\mathrm{SEP}_{\ell}$ does not contain any random variables, it can certainly be calculated numerically using software packages such as MATLAB.

\section{Optimal Power Calculation Method for the FD Mode}

Since the system performance depends much on the RSI, finding the optimal power for the transmit power at the FD relay node is important. In addition to the reduction of RSI, the optimal power also helps to save power for the relay node. Therefore, in this section, we propose an optimal power calculation method for the FD mode to select the transmit power $P_{\mathrm{R}}$ according to the SIC capability $\left(\tilde{\Omega}_{\mathrm{R}}\right)$, the transmit power of the source node $\left(P_{1}\right)$, the hardware impairments $\left(k_{\mathrm{S}_{1} \mathrm{R}}\right.$ and $\left.k_{\mathrm{RS}}\right)$, the distance between the nodes $\left(\Omega_{1}, \Omega_{2}, \Omega_{3}\right)$, the threshold $(x)$, and the AWGN at the destination node $\left(\sigma_{2}^{2}\right)$. The optimal transmit power that is optimized with respect to minimum OP of the system is derived in the following sections.

4.1. Case 1: Without Direct Link. The optimization problem for the relay transmit power of the FD mode can be formulated as follows:

$$
\begin{aligned}
& \min \mathrm{OP}^{(1)-\text { ex }} \\
& \text { s.t. } P_{\mathrm{R}}>0 \text { and } x<d .
\end{aligned}
$$

In order to solve this problem, we can use a linear programming method to get the closed-form expression of the relay optimal power, denoted by $P_{\mathrm{R}}^{*}$, as follows:

$$
P_{\mathrm{R}}^{*}=\arg \min _{P_{\mathrm{R}}} \mathrm{OP}^{(1) \_ \text {ex }},
$$

where $\mathrm{OP}^{(1) \_e x}$ is determined using (18). The power calculation method for Case 1 is summarized in Algorithm 1.

Note that when the system has no optimal power, i.e., $P_{\mathrm{R}}^{*}=\varnothing$, choose $P_{\mathrm{R}}$ based on the resulting expression of $\partial \mathrm{O}$ $\mathrm{P}^{(1) \_e x} / \partial P_{\mathrm{R}}$; herein, $\varnothing$ denotes the empty set.

Taking the derivative of $\mathrm{OP}^{(1) \_ \text {ex }}$ with respect to $P_{\mathrm{R}}$ gives us

$$
\begin{aligned}
\frac{\partial \mathrm{OP}^{(1)_{-} \mathrm{ex}}}{\partial P_{\mathrm{R}}}= & -\exp \left(-\frac{\left(\sigma_{\mathrm{R}}^{2}+\tilde{\Omega}_{\mathrm{R}} P_{\mathrm{R}}\right) x}{\Omega_{1} P_{1}\left(1-k_{\mathrm{S}_{1} \mathrm{R}}^{2} x\right)}-\frac{\sigma_{2}^{2} x}{\Omega_{2} P_{\mathrm{R}}\left(1-k_{\mathrm{RS}_{2}}^{2} x\right)}\right) \\
& \times\left[-\frac{\tilde{\Omega}_{\mathrm{R}} x}{\Omega_{1} P_{1}\left(1-k_{\mathrm{S}_{1} \mathrm{R}}^{2} x\right)}+\frac{\sigma_{2}^{2} x}{\Omega_{2} P_{\mathrm{R}}^{2}\left(1-k_{\mathrm{RS}_{2}}^{2} x\right)}\right] .
\end{aligned}
$$

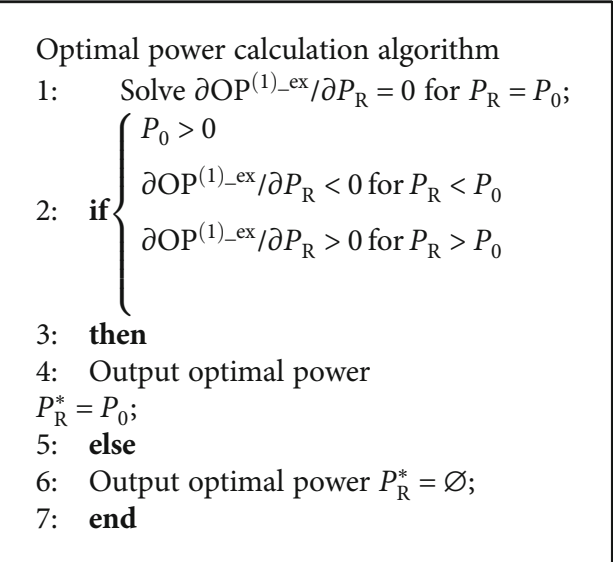

Algorithm 1:

From (40), it can be easily shown that $\partial \mathrm{OP}^{(1)}{ }^{\mathrm{ex}} / \partial P_{\mathrm{R}}=0$ when $\left.P_{\mathrm{R}}=P_{0}, \partial \mathrm{OP}^{(1)}\right)_{\text {ex }} / \partial P_{\mathrm{R}}<0$ when $P_{\mathrm{R}}<P_{0}$, and $\partial \mathrm{O}$ $\mathrm{P}^{(1) \_ \text {ex }} / \partial P_{\mathrm{R}}>0$ when $P_{\mathrm{R}}>P_{0}$. Therefore, $P_{\mathrm{R}}^{*}$ is the optimal transmit power of the relay node, which is given by

$$
P_{\mathrm{R}}^{*}=P_{0}=\sqrt{\frac{\Omega_{1} P_{1} \sigma_{2}^{2}\left(1-k_{\mathrm{S}_{1} \mathrm{R}}^{2} x\right)}{\Omega_{2} \tilde{\Omega}_{\mathrm{R}}\left(1-k_{\mathrm{RS}_{2}}^{2} x\right)}} .
$$

4.2. Case 2: With the Direct Link but No Cooperative Communication. Similar to Case 1, we can create a similar algorithm as Algorithm 1 by replacing $\mathrm{OP}^{(1) \_\mathrm{ex}}$ by $\mathrm{OP}^{(2) \_\mathrm{ex}}$. The calculation method for the optimal power for Case 2 can be summarized as follows. Solving $\partial \mathrm{OP}^{(2) \_\mathrm{ex}} / \partial P_{\mathrm{R}}=0$ (for the case $x<d$ ) results in the following equation:

$$
A P_{\mathrm{R}}^{3}+B P_{\mathrm{R}}^{2}+C P_{\mathrm{R}}+D=0,
$$

and the optimal power $P_{\mathrm{R}}^{*}$ is given by

$$
P_{\mathrm{R}}^{*}=\frac{2 \sqrt{\Delta} \cos (\arccos (K) / 3)-B}{3 A},
$$

where $A=\Omega_{2}^{2}\left(1-k_{\mathrm{RS}}^{2} x\right)^{2} \tilde{\Omega}_{\mathrm{R}} x, \quad B=\Omega_{2}\left(1-k_{\mathrm{RS}}^{2} x\right) \Omega_{3} P_{1}(1+$ $\left.k_{\mathrm{S}_{1} \mathrm{R}}^{2}\right) \tilde{\Omega}_{\mathrm{R}} x^{2}, \quad C=-\Omega_{1} P_{1}\left(1-k_{\mathrm{S}_{1} \mathrm{R}}^{2} x\right) \Omega_{2}\left(1-k_{\mathrm{RS}}^{2} x\right)\left[\Omega_{3} P_{1}(1+\right.$ $\left.\left.k_{\mathrm{S}_{1} \mathrm{R}}^{2}\right) x+\sigma_{2}^{2} x\right], D=-\Omega_{1} P_{1}\left(1-k_{\mathrm{S}_{1} \mathrm{R}}^{2} x\right) \Omega_{3} P_{1}\left(1+k_{\mathrm{S}_{1} \mathrm{R}}^{2}\right) \sigma_{2}^{2} x^{2}, \Delta=$ $B^{2}-3 A C$, and

$$
K=\frac{9 A B C-2 B^{3}-27 A^{2} D}{2 \sqrt{\Delta^{3}}} .
$$

4.3. Case 3: With the Direct Link and Cooperative Communication. Similar to the above method, in this case, for the first case $(x<d, G(x) \neq 0)$ of equation (27), due to the 


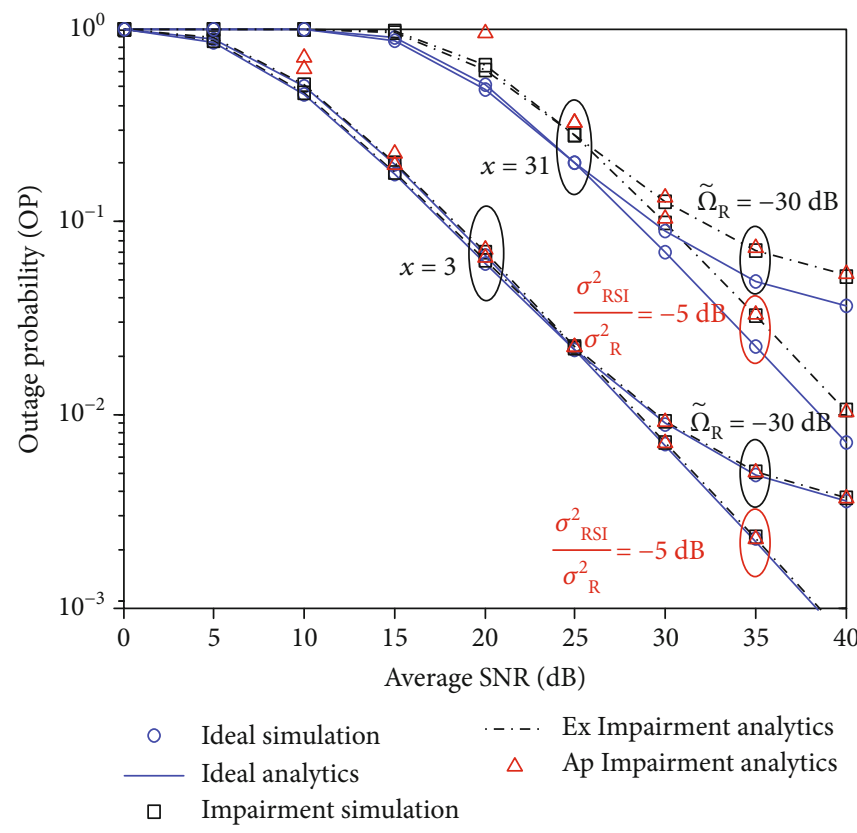

FIGURE 2: OP of the system for the case without the direct link. $\Omega_{1}=\Omega_{2}=1, \sigma_{\mathrm{R}}^{2}=\sigma_{2}^{2}=1$, and $k_{\mathrm{S}_{1} \mathrm{R}}=k_{\mathrm{RS}}=0.1$. Fixed and varied RSI as $\sigma_{\mathrm{RSI}}^{2} / \sigma_{\mathrm{R}}^{2}=-5 \mathrm{~dB}$ and $\tilde{\Omega}_{\mathrm{R}}=-30 \mathrm{~dB}$, respectively. $\mathscr{R}=2$ and $5 \mathrm{bits} / \mathrm{s} / \mathrm{Hz}$.

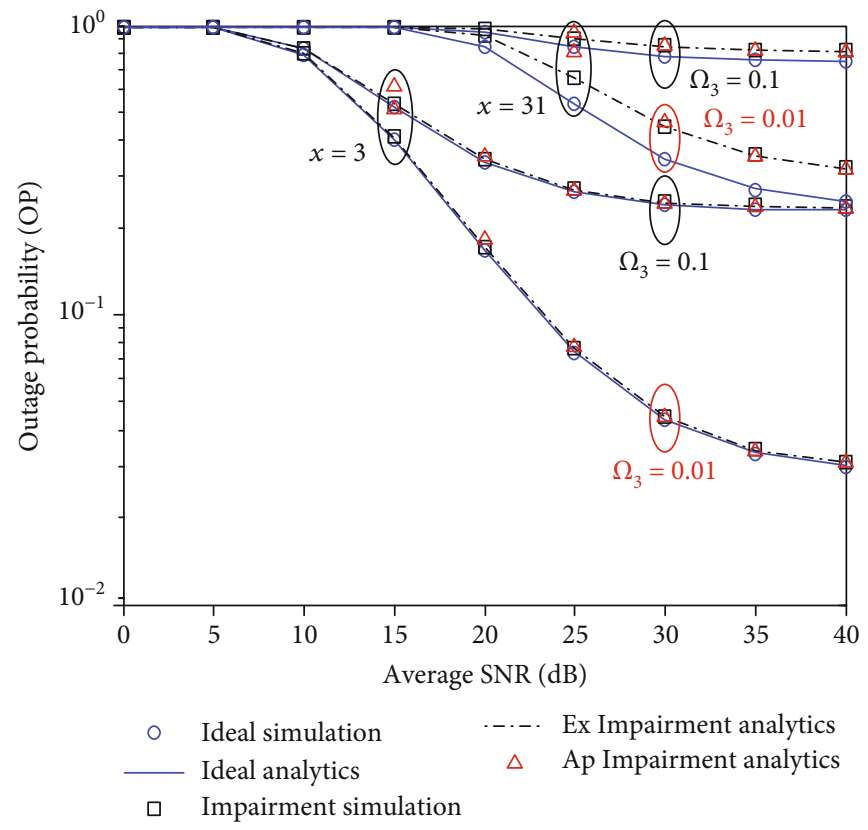

FIGURE 3: OP of the system for the case with the direct link but without cooperation. $\Omega_{1}=\Omega_{2}=1$ and $\sigma_{\mathrm{R}}^{2}=\sigma_{2}^{2}=1 . k_{\mathrm{S}_{1} \mathrm{R}}=k_{\mathrm{RS}}=0.1$ and $\sigma_{\mathrm{RSI}}^{2} / \sigma_{\mathrm{R}}^{2}=5 \mathrm{~dB}$. Two power levels of interference: $\Omega_{3}=0.01$ and $\Omega_{3}=0.1 . \mathscr{R}=2$ and $5 \mathrm{bits} / \mathrm{s} / \mathrm{Hz}$.

complexity of the OP, it is not possible to find the critical points. However, we can find the suboptimal points using some mathematical manipulations. In this case, the OP can be rewritten as follows:

$$
\mathrm{OP}^{(3) \_\mathrm{ex}}=1-\frac{e^{-\alpha_{1}(x)-\alpha_{2}(x)}}{a-b}\left(a-b e^{\alpha_{2}(x)-\alpha_{3}(x)}\right) \text {, }
$$

where $a=\Omega_{2} P_{\mathrm{R}}\left(1-k_{\mathrm{RS}_{2}}^{2} x\right)$ and $b=\Omega_{3} P_{1}\left(1-k_{\mathrm{S}_{1} \mathrm{R}}^{2} x\right)$. For $a>$ $b$, we have $\alpha_{2}(x)<\alpha_{3}(x)$; thus, $e^{\alpha_{2}(x)-\alpha_{3}(x)}<1$. Therefore, $a-$ $b e^{\alpha_{2}(x)-\alpha_{3}(x)}>0$. So, the suboptimal point is the one that maximizes $e^{-\alpha_{1}(x)-\alpha_{2}(x)}$. From here, we get the suboptimal power $P_{\mathrm{R}}^{*}$ as given by (41). For $a<b$, using the similar method, we can also get the suboptimal power $P_{\mathrm{R}}^{*}$ as in (41). As a result, for the first case of (27), $P_{\mathrm{R}}^{*}$ is 


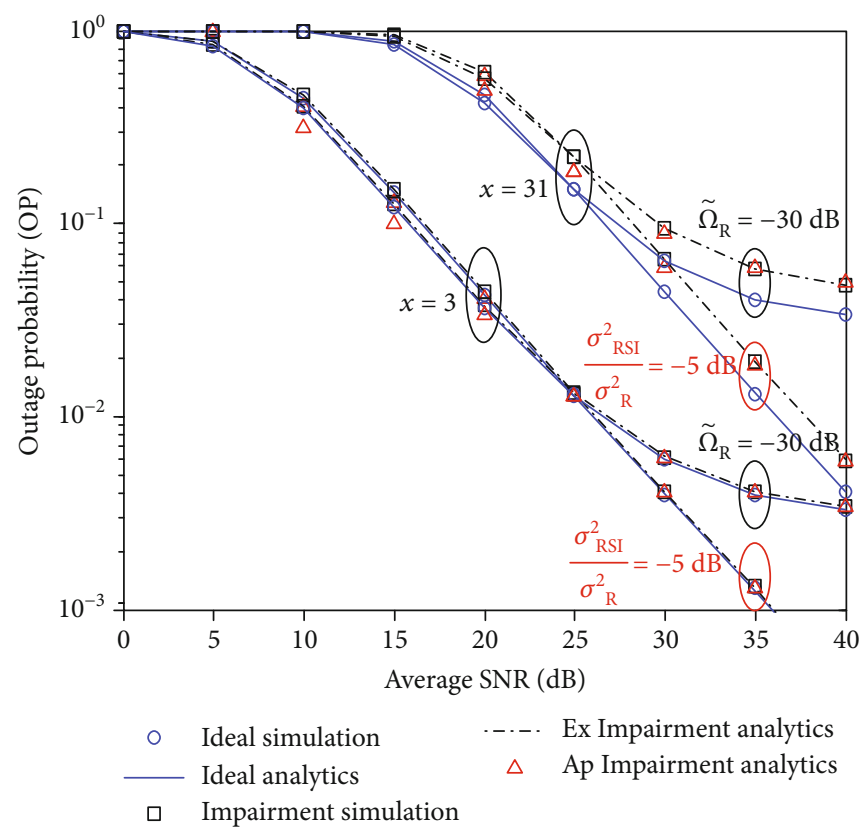

FIGURE 4: OP for the case with the direct link and using cooperation. $\Omega_{1}=\Omega_{2}=1$ and $\sigma_{\mathrm{R}}^{2}=\sigma_{2}^{2}=1 . k_{\mathrm{S}_{1} \mathrm{R}}=k_{\mathrm{RS}_{2}}=0.1$ and $\Omega_{3}=0.1$. Fixed and varied RSI as $\sigma_{\mathrm{RSI}}^{2} / \sigma_{\mathrm{R}}^{2}=-5 \mathrm{~dB}$ and $\tilde{\Omega}_{\mathrm{R}}=-30 \mathrm{~dB}$, respectively. $\mathscr{R}=2$ and $5 \mathrm{bits} / \mathrm{s} / \mathrm{Hz}$.

determined by (41). For the second case $(x<d, G(x)=0)$, there is only one $P_{\mathrm{R}}$ that makes $G(x)=0$. For the third case $\left(1 / k_{\mathrm{RS}_{2}}^{2} \leqslant x<1 / k_{\mathrm{S}_{1} \mathrm{R}}^{2}\right)$, the OP of the system is minimized when $P_{\mathrm{R}}$ approaches zero.

\section{Performance Evaluation and Discussions}

In this section, we present performance evaluation of the system using numerical calculation. Monte Carlo simulations using generated random channels are also used to verify our theoretical analysis. In our simulations, we set $\Omega_{1}=\Omega_{2}=1$ and $\sigma_{\mathrm{R}}^{2}=\sigma_{2}^{2}=\sigma^{2}=1$. The average SNR is defined as $\operatorname{SNR}=P_{1} / \sigma^{2}$. In the case of no optimization, the transmit power at the relay is set as $P_{\mathrm{R}}=P_{1}$. In the case of applying optimal transmit power, $P_{\mathrm{R}}=P_{1}$ is computed as shown in Section 4. In order to obtain the OP, two thresholds are used for the OP, which are $x=2^{2}-1=3$ and $x=$ $2^{5}-1=31$ with the minimum required data rates of 2 bits/s/Hz and 5 bits/s/Hz, respectively. To verify our theoretical analysis, simulated results are also plotted together with numerical ones for comparison.

Figure 2 shows the OP for the case without the direct link from the source to the destination, i.e., Case 1. The plotted curves were obtained by numerical calculations using equations (18), (19), and (32), while the marker symbols by computer simulations with $k_{\mathrm{S}_{1} \mathrm{R}}=k_{\mathrm{RS}_{2}}=0.1$, $\Omega_{1}=\Omega_{2}=1$, and $\sigma_{\mathrm{R}}^{2}=\sigma_{2}^{2}=1$. The RSI was considered for two cases: (i) fixed at $\sigma_{\mathrm{RSI}}^{2} / \sigma_{\mathrm{R}}^{2}=-5 \mathrm{~dB}$ and (ii) varying with transmit power of the relay node $\left(\sigma_{\mathrm{RSI}}^{2}=\tilde{\Omega}_{\mathrm{R}} P_{\mathrm{R}}\right.$ with $\tilde{\Omega}_{\mathrm{R}}=$ $-30 \mathrm{~dB}$ ). Note that in the first case, the level of RSI is intentionally set smaller than the noise variance to bypass the RSI effect and explore the system performance under the

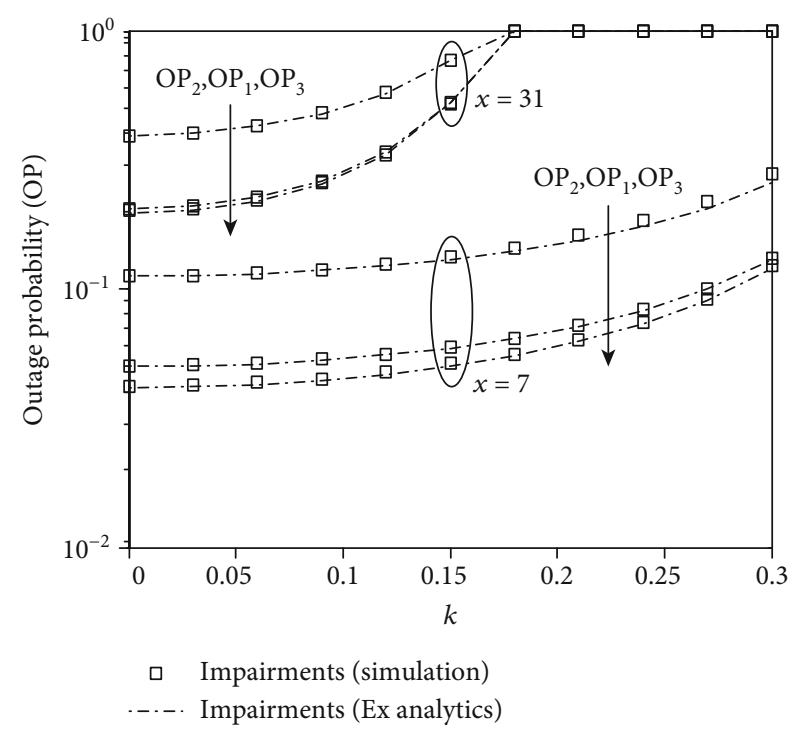

FIgURE 5: OP for the three cases versus the distortion factor $k$. $\mathrm{SNR}=25 \mathrm{~dB}, \sigma_{\mathrm{RSI}}^{2} / \sigma_{\mathrm{R}}^{2}=-5 \mathrm{~dB}, x=31$ and 7 , and $\Omega_{3}=0.01$.

impact of only HI. As can be seen from the figure when the threshold is low $(x=3)$, the performance loss due to hardware impairments is very small and can be neglected. However, when the threshold becomes larger $(x=31)$, this loss becomes significant and cannot be overlooked. This means that the effect of hardware impairments on the system quality is more significant in a high-rate system than in a low-rate one. Therefore, employed devices in the high-rate systems should be fabricated with high precision in order to avoid unnecessary performance loss. It is also noticed from the figure that when the RSI varies with the transmit power 


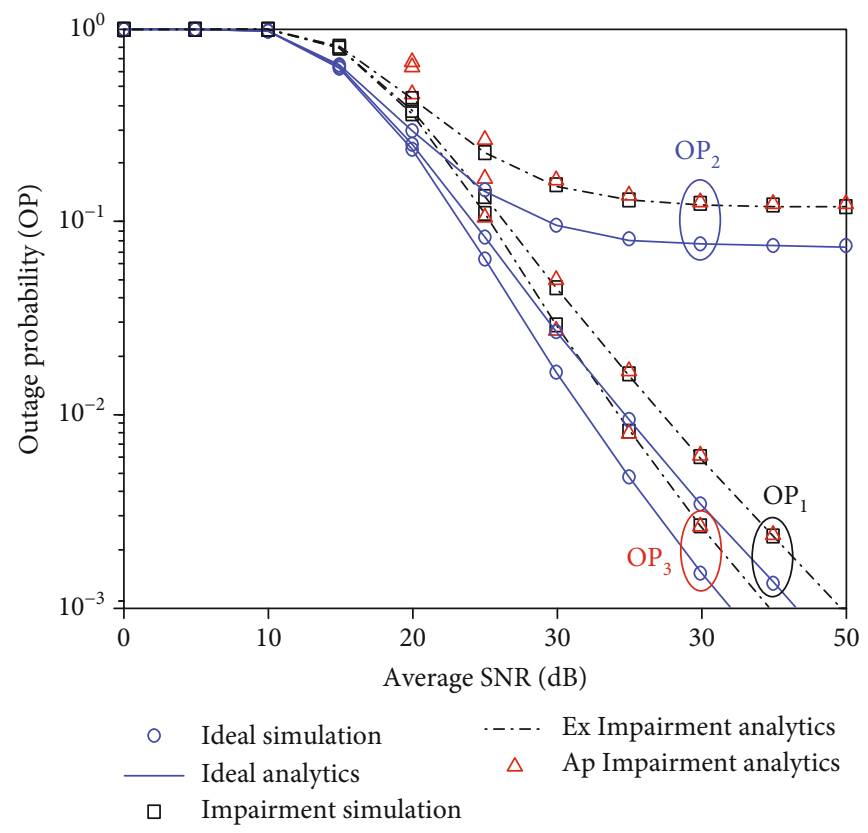

FIGURE 6: OP performance for the case with the optimal transmit power. $k_{\mathrm{S}_{1} \mathrm{R}}=k_{\mathrm{RS}_{2}}=0.1, x=31, \tilde{\Omega}_{\mathrm{R}}=-30 \mathrm{~dB}$, and $\Omega_{3}=0.01$ for OP 2 and $\Omega_{3}=0.1$ for $\mathrm{OP}_{3}$.

of the relay node, the OP performance is significantly affected especially at the high SNR region.

Figure 3 shows the achieved OP for the case with the direct link but without cooperation (Case 2) using analytical equations (24), (25), and (33) with $\sigma_{\mathrm{RSI}}^{2} / \sigma_{\mathrm{R}}^{2}=5 \mathrm{~dB}$ and the distortion factors $k_{\mathrm{S}_{1} \mathrm{R}}=k_{\mathrm{RS}_{2}}=0.1$ and $\Omega_{1}=\Omega_{2}=1$. Since cooperation was not used, the signal from the source node to the destination node was regarded as interference. Two specific power levels of interference, namely, $\Omega_{3}=0.01$ and $\Omega_{3}=0.1$, were used for investigation. Compared with the previous case, the outage performance of the system is seriously degraded, especially when the interference level is high. Note that for this level of interference, the OP performance will not increase when the RSI decreases, i.e., $\sigma_{\mathrm{RSI}}^{2} / \sigma_{\mathrm{R}}^{2}=-5$ $\mathrm{dB}$. Moreover, the system suffers from an irreducible outage floor at the high SNR region. When it is possible, cooperation can be used with an appropriate combining scheme such as Case 3 to alleviate the effect of this interference and improve the system performance.

Figure 4 shows the OP for the case using cooperation (Case 3) with $\Omega_{3}=0.1$. Other parameters for numerical calculation are the same as in Figure 2. We can see from the figure that although cooperation via the direct link is used, the ideal system achieves only diversity order 1 as in the case without cooperation in Figure 2 since DF was used for relaying. However, the system with HI benefits significantly from the cooperation which allows for performance improvement. Compared with those shown in Figures 2 and 3, the system achieves the best performance thanks to cooperation.

Figure 5 illustrates the impact of the distortion factor $k$ on the outage performance of the system for the abovementioned three cases, i.e., without the direct link $\left(\mathrm{OP}_{1}\right)$,

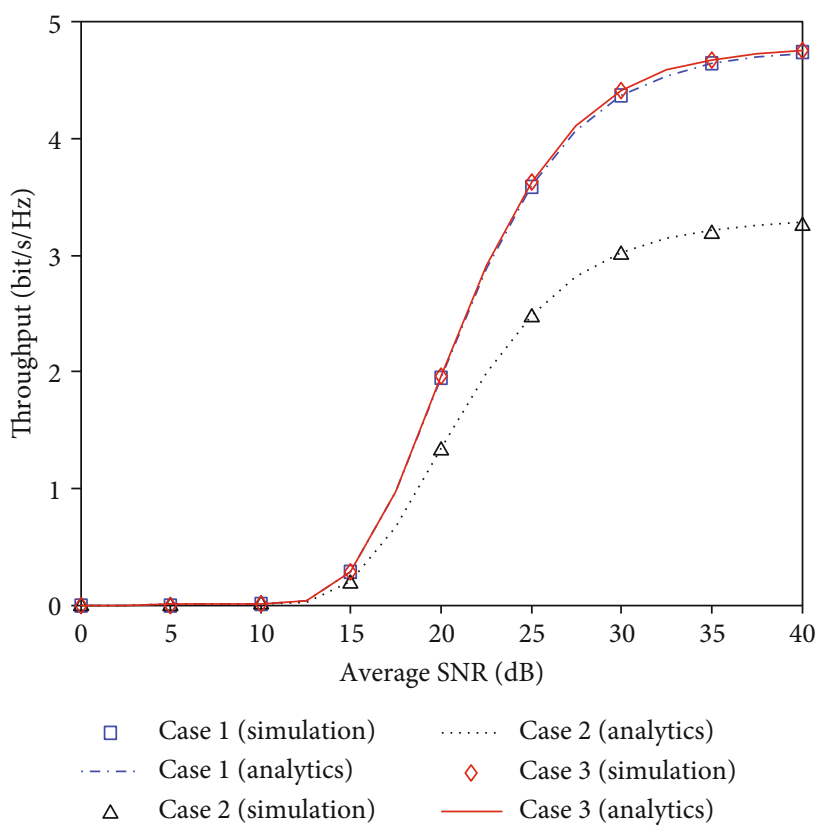

FIgURE 7: System throughput for the three cases using the optimal power values. $\Omega_{1}=\Omega_{2}=1, \sigma_{\mathrm{R}}^{2}=\sigma_{2}^{2}=1 . k_{\mathrm{S}_{1} \mathrm{R}}=k_{\mathrm{RS}_{2}}=0.1, \Omega_{3}=0.01$, and $\tilde{\Omega}_{\mathrm{R}}=-30 \mathrm{~dB}$.

with the direct link but without cooperation $\left(\mathrm{OP}_{2}\right)$, and with the direct link and with cooperation $\left(\mathrm{OP}_{3}\right)$. The average SNR at the relay node and the destination node were both set to $25 \mathrm{~dB}, \sigma_{\mathrm{RSI}}^{2} / \sigma_{\mathrm{R}}^{2}=-5 \mathrm{~dB}$, and $\Omega_{3}=0.01$. Two values of $x$ were used for investigation, namely, $x=31$ and $x=7$. As can be seen from the figure, the impact of hardware impairments for all the three cases is trivial for small distortion factor $x$ 


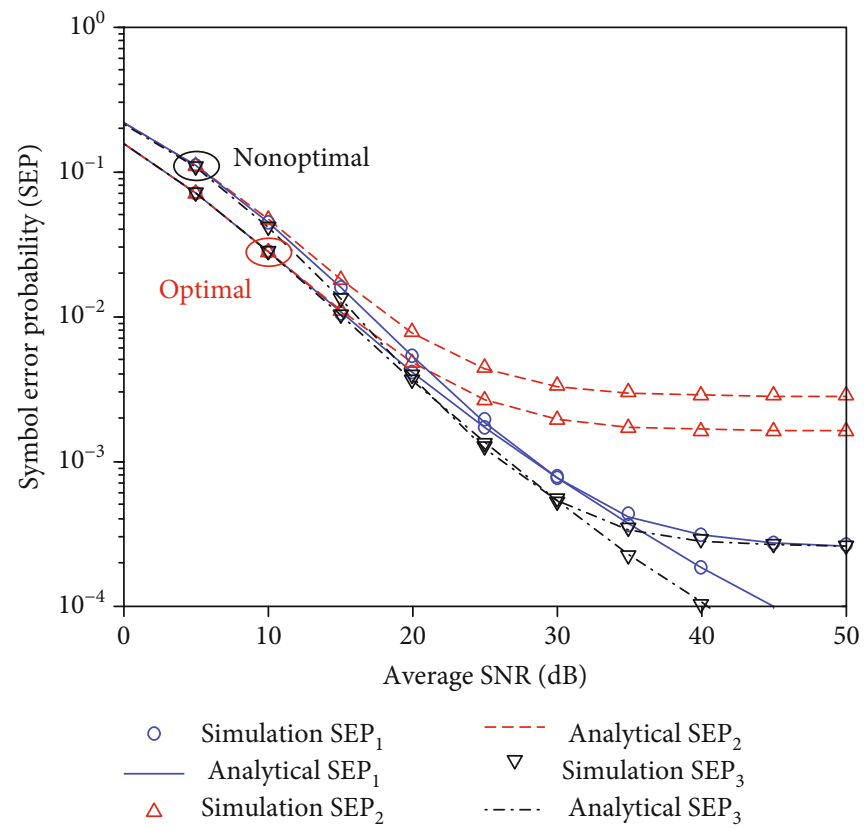

FIGURE 8: Effect of hardware impairment on the SEP performance with and without the optimal power values. $k_{\mathrm{S}_{1} \mathrm{R}}=k_{\mathrm{RS}_{2}}=0.1 . \Omega_{3}=0.01$ and $\tilde{\Omega}_{\mathrm{R}}=-30 \mathrm{~dB}$.

but becomes more significant as it increases. The system with a higher transmission rate is more influenced by hardware impairments than that with a lower one. For $x=31$, the system soon experiences link outage with probability one at $k=0.17$. The system with a lower rate $(x=7)$, however, can resist OP less than 0.1 within the acceptable hardware impairment range. In order to reduce OP, the optimal power needs to be used to reduce the impact of the RSI. On the other hand, reducing transmit power can also help to decrease the distortion factor $k$ because hardware impairments depend on the amplifier operation.

Figure 6 shows the OP versus the average SNR at the relay node when optimal power values are used. In this figure, the transmit power at the FD node was set using equations (41) for $\mathrm{OP}_{1}$ and $\mathrm{OP}_{3}$ and (43) for $\mathrm{OP}_{2}$. The system was set up with $x=31$ and $\tilde{\Omega}_{\mathrm{R}}=-30 \mathrm{~dB}$ while the remaining parameters are used as shown in Figure 2. The $\mathrm{OP}_{2}$ curve was obtained with $\Omega_{3}=0.01$ and the $\mathrm{OP}_{3}$ curve with $\Omega_{3}=0.1$. Comparing Figures 2-4 for the case in which the RSI is varied by the transmit power of the FD node, we can see clearly that the optimal power values can increase the outage performance of the system with hardware impairments significantly. Although the outage floor still exists for Case 2 at SNR > 30 $\mathrm{dB}$, it is completely avoided for Cases 1 and 3 .

Figure 7 plots the throughput of the systems as a function of the average SNR at the relay node for the three cases when $\mathscr{R}=5 \mathrm{bits} / \mathrm{s} / \mathrm{Hz}, \Omega_{3}=0.01$, and $\tilde{\Omega}_{\mathrm{R}}=-30 \mathrm{~dB}$. As can be seen in the figure, for high-rate systems, the throughput reaches the target rate $\mathscr{R}$ at $\mathrm{SNR}>40 \mathrm{~dB}$ for Cases 1 and 3 . For Case 2 , due to the effect of interference, the throughput cannot reach the target rate even when using self-interference cancellation.
The SEP of the system using BPSK modulation and the same assumptions used in Figure 2 is analyzed as shown in Figure 8. Other parameters were as follows: $\Omega_{3}=0.01$ and $\tilde{\Omega}_{\mathrm{R}}=-30 \mathrm{~dB}$. The analytical curves were obtained using equation (37) by using MATLAB. Denoted by $\mathrm{SEP}_{1}, \mathrm{SEP}_{2}$, and $\mathrm{SEP}_{3}$ are the SEPs of the system for Cases 1, 2, and 3, respectively. The SEPs are shown as a function of the average SNR at the relay and the destination node with and without the optimal power. It is clear that the derived optimal power values help to increase the SEP performance of the system, especially for Cases 1 and 3.

\section{Conclusion}

In this paper, we analyzed the system performance of the IBFD DF relay systems with hardware impairments and imperfect self-interference cancellation. We derived exact and approximate expressions of $\mathrm{OP}$, as well as its asymptotic values at high signal power. In order to alleviate the effect of RSI, we have proposed an optimal power calculation method based on the minimum OP for the case without the direct link and with the direct link but without cooperation. For the case of a direct link with cooperation, the proposed scheme still works but yields suboptimal solutions. Various performance evaluations have been conducted to investigate the effect of hardware impairments and the RSI on the system behavior. The evaluated results show that the high-rate system is more vulnerable to the hardware impairments than the low-rate one. However, using the proposed optimal power value can reduce the effect of hardware impairments and imperfect SIC. Moreover, for the IBFD DF relay networks, it is recommended that cooperative communication 
should be used for the case with the direct link in order to avoid the irreducible outage floor. Also, although our analysis and optimal power calculation can be extended to the case of multiple-relay networks, the impact of superposed hardware impairments and RSI together with the disappearance of the direct link makes it more difficult for these networks to cope with the early performance saturation issue. Thus, more effective RSI cancellation solutions and high-quality hardware devices should be employed when considering the IBFD communication for the multiple-relay networks.

\section{Data Availability}

The simulation data used to support the findings of this study are included within the article.

\section{Disclosure}

The work by Ba Cao Nguyen, Xuan Nam Tran, and Thi Thu Hang Nguyen was performed as part of the employment in Le Quy Don Technical University, and that by Ba Cao Nguyen and Dinh Tan Tran as part of the employment in Telecommunications University.

\section{Conflicts of Interest}

The authors declare that they have no conflicts of interest.

\section{References}

[1] A. H. Gazestani, S. A. Ghorashi, B. Mousavinasab, and M. Shikh-Bahaei, "A survey on implementation and applications of full duplex wireless communications," Physical Communication, vol. 34, pp. 121-134, 2019.

[2] B. C. Nguyen, X. N. Tran, T. M. Hoang, and L. T. Dung, "Performance analysis of full-duplex vehicle-to-vehicle relay system over double-Rayleigh fading channels," Mobile Networks and Applications, vol. 25, no. 1, pp. 363-372, 2020.

[3] B. C. Nguyen, T. M. Hoang, and P. T. Tran, "Performance analysis of full-duplex decode-and-forward relay system with energy harvesting over Nakagami- $m$ fading channels," $A E U$ International Journal of Electronics and Communications, vol. 98, pp. 114-122, 2019.

[4] C. Li, Z. Chen, Y. Wang, Y. Yao, and B. Xia, "Outage analysis of the full-duplex decode-and-forward two-way relay system," IEEE transactions on Vehicular Technology, vol. 66, no. 5, pp. 4073-4086, 2017.

[5] M. K. Fikadu, P. C. Sofotasios, M. Valkama, S. Muhaidat, Q. Cui, and G. K. Karagiannidis, "Outage probability analysis of dual-hop full-duplex decode-and-forward relaying over generalized multipath fading conditions," in 2015 IEEE 11th International Conference on Wireless and Mobile Computing, Networking and Communications (WiMob), pp. 414-421, Abu Dhabi, 2015.

[6] L. Li, C. Dong, L. Wang, and L. Hanzo, "Spectral-efficient bidirectional decode-and-forward relaying for full-duplex communication," IEEE Transactions on Vehicular Technology, vol. 65, no. 9, pp. 7010-7020, 2016.

[7] B. C. N. Le Van Nguyen, X. N. Tran, and L. T. Dung, "Transmit antenna selection for full-duplex spatial modulation multiple-input multiple-output system," IEEE Systems Journal, 2020.

[8] K. Guo, B. Zhang, Y. Huang, and D. Guo, "Outage analysis of multi-relay networks with hardware impairments using SECps scheduling scheme in shadowed-Rician channel," IEEE Access, vol. 5, pp. 5113-5120, 2017.

[9] C. Li, H. Wang, Y. Yao, Z. Chen, X. Li, and S. Zhang, "Outage performance of the full-duplex two-way DF relay system under imperfect CSI," IEEE Access, vol. 5, pp. 5425-5435, 2017.

[10] C. Li, B. Xia, S. Shao, Z. Chen, and Y. Tang, "Multi-user scheduling of the full-duplex enabled two-way relay systems," IEEE Transactions on Wireless Communications, vol. 16, no. 2, pp. 1094-1106, 2017.

[11] X. N. Tran, B. C. Nguyen, and D. T. Tran, "Outage probability of two-way full-duplex relay system with hardware impairments," in 2019 3rd International Conference on Recent Advances in Signal Processing, Telecommunications \& Computing (SigTelCom), pp. 135-139, Hanoi, Vietnam, Vietnam, 2019.

[12] G. I. Tsiropoulos, A. Yadav, M. Zeng, and O. A. Dobre, "Cooperation in $5 \mathrm{G}$ hetnets: advanced spectrum access and D2D assisted communications," IEEE Wireless Communications, vol. 24, no. 5, pp. 110-117, 2017.

[13] B. C. Nguyen, X. N. Tran, D. T. Tran, and L. T. Dung, "Fullduplex amplify-and-forward relay system with direct link: performance analysis and optimization," Physical Communication, vol. 37, article 100888, 2019.

[14] A. Sabharwal, P. Schniter, D. Guo, D. W. Bliss, S. Rangarajan, and R. Wichman, "In-band full-duplex wireless: challenges and opportunities," IEEE Journal on selected areas in communications, vol. 32, no. 9, pp. 1637-1652, 2014.

[15] B. C. Nguyen, X. N. Tran, and D. T. Tran, "Performance analysis of in-band full-duplex amplify-and-forward relay system with direct link," in 2018 2nd International Conference on Recent Advances in Signal Processing, Telecommunications \& Computing (SigTelCom), pp. 192-197, Ho Chi Minh City, Vietnam, 2018.

[16] A. Omri, A. S. Behbahani, A. M. Eltawil, and M. O. Hasna, "Performance analysis of full-duplex multiuser decode-andforward relay networks with interference management," in 2016 IEEE Wireless Communications and Networking Conference, pp. 1-6, Doha, Qatar, April 2016.

[17] K. C. Dheeraj, A. Thangaraj, and R. Ganti, "Equalization in amplify-forward full-duplex relay with direct link," in 2015 Twenty First National Conference on Communications (NCC), pp. 1-6., Mumbai, India, 2015.

[18] Y. Wang, Y. Xu, N. Li, W. Xie, K. Xu, and X. Xia, "Relay selection of full-duplex decode-and-forward relaying over Nakagami-m fading channels," IET Communications, vol. 10, no. 2, pp. 170-179, 2016.

[19] N. E. Wu and H. J. Li, "Performance analysis of SNR-based decode-and-forward opportunistic relaying in the presence of cochannel interference," IEEE Transactions on Vehicular Technology, vol. 65, no. 9, pp. 7244-7257, 2016.

[20] E. Bjornson, M. Matthaiou, and M. Debbah, "A new look at dual-hop relaying: performance limits with hardware impairments," IEEE Transactions on Communications, vol. 61, no. 11, pp. 4512-4525, 2013.

[21] A. K. Mishra, S. C. M. Gowda, and P. Singh, "Impact of hardware impairments on TWRN and OWRN AF relaying systems 
with imperfect channel estimates," in 2017 IEEE Wireless Communications and Networking Conference (WCNC), pp. 1-6, San Francisco, CA, USA, March 2017.

[22] Y. Liu, X. Xue, J. Zhang, X. Li, L. Dai, and S. Jin, "Multipair massive MIMO two-way full-duplex relay systems with hardware impairments," in GLOBECOM 2017-2017 IEEE Global Communications Conference, pp. 1-6, Singapore, Singapore, 2017.

[23] S. Dey, E. Sharma, and R. Budhiraja, "Scaling analysis of hardware-impaired two-way full-duplex massive MIMO relay," IEEE Communications Letters, vol. 23, no. 7, pp. 1249-1253, 2019.

[24] O. Taghizadeh, A. C. Cirik, and R. Mathar, "Hardware impairments aware transceiver design for full-duplex amplify-andforward MIMO relaying," IEEE Transactions on Wireless Communications, vol. 17, no. 3, pp. 1644-1659, 2018.

[25] B. C. Nguyen, N. N. Thang, X. N. Tran, and L. T. Dung, "Impacts of imperfect channel state information, transceiver hardware, and self-interference cancellation on the performance of full-duplex mimo relay system," Sensors, vol. 20, no. 6, 2020.

[26] O. Taghizadeh, V. Radhakrishnan, A. C. Cirik, R. Mathar, and L. Lampe, "Hardware impairments aware transceiver design for bidirectional full-duplex MIMO OFDM systems," IEEE Transactions on Vehicular Technology, vol. 67, no. 8, pp. 7450-7464, 2018.

[27] B. C. Nguyen, T. M. Hoang, and P. T. Tran, "Improving the performance of spatial modulation full-duplex relaying system with hardware impairment using transmit antenna selection," IEEE Access, vol. 8, pp. 20191-20202, 2020.

[28] T. Schenk, RF imperfections in high-rate wireless systems: impact and digital compensation, $1 \mathrm{em}$ plus $0.5 \mathrm{em}$ minus 0.4em Springer Science \& Business Media, 2008.

[29] C. Studer, M. Wenk, and A. Burg, "MIMO transmission with residual transmit-RF impairments," in 2010 International ITG Workshop on Smart Antennas (WSA), pp. 189-196, Bremen, Germany, 2010.

[30] A. Papazafeiropoulos, S. K. Sharma, T. Ratnarajah, and S. Chatzinotas, "Impact of residual additive transceiver hardware impairments on Rayleigh-product MIMO channels with linear receivers: exact and asymptotic analyses," IEEE Transactions on Communications, vol. 66, no. 1, pp. 105-118, 2018.

[31] D. Bharadia, E. McMilin, and S. Katti, "Full duplex radios," in Proceedings of the ACM SIGCOMM 2013 conference on SIGCOMM, pp. 375-386, New York, NY, USA, 2013.

[32] A. Hyadi, M. Benjillali, and M. S. Alouini, "Outage performance of decode-and-forward in two-way relaying with outdated CSI," IEEE Transactions on Vehicular Technology, vol. 64, no. 12, pp. 5940-5947, 2015.

[33] B. C. Nguyen and X. N. Tran, "Performance analysis of fullduplex amplify-and-forward relay system with hardware impairments and imperfect self-interference cancellation," Wireless Communications and Mobile Computing, vol. 2019, Article ID 4946298, 10 pages, 2019.

[34] A. Leon-Garcia, Probability, statistics, and random processes for electrical engineering, $1 \mathrm{em}$ plus $0.5 \mathrm{em}$ minus $0.4 \mathrm{em}$ Pearson/Prentice Hall, Upper Saddle River, NJ, 3rd edition, 2008.

[35] A. Goldsmith, Wireless communications, $1 \mathrm{em}$ plus $0.5 \mathrm{em}$ minus 0.4em Cambridge university press, 2005. 